\title{
On Vibrations Of An Axially Moving Beam Under Material Damping
}

\author{
Sajad H. Sandilo ${ }^{1,3}$, Rajab A. Malookani ${ }^{2}$, and Abdul Hanan Sheikh ${ }^{1}$ \\ ${ }^{1}$ Department of Mathematics and Statistics, Faculty of Science, Quaid-e-Awam University of Engineering, \\ Science and Technology, 67480 Nawabshah, Sindh, Pakistan \\ ${ }^{2}$ Mathematical Physics Group, Delft Institute of Applied Mathematics, Faculty of Electrical Engineering, \\ Mathematics and Computer Science, Delft University of Technology, 2628CD Delft, The Netherlands \\ ${ }^{3}$ Corresponding author (SH Sandilo, email: s.h.sandilo@quest.edu.pk)
}

\begin{abstract}
In this paper a materially damped linear homogeneous beam-like equation has been considered. The viscoelastic beam is simply supported at both ends, whereas general initial conditions are considered. From mechanical and physical point of view the problem describes a mathematical model of internally damped transversal vibrations of a moving conveyor belt or a viscoelastic chain drive. From Hamilton's principle, a fifth order partial differential equation (PDE) for axially moving continuum has been formulated. The axial speed of the beam is considered to be positive, constant and small compared to wave velocity, and it is also assumed that the introduced material damping is relatively small. The solutions of equation of motion are based upon a two timescales perturbation method. By application of this perturbation method, it has been shown that the material damping does in fact affect the solution responses, and it reduces the vibration and noise in the system. It has also been shown that the material damping generated in the system depends on the mode number $n$, which is obviously expected from mechanical point of view.
\end{abstract}

Keywords: Conveyor belt, Beam-like, Axially moving, Material damping, Two timescales

\section{Introduction}

In world around us, almost all physical, structural, and mechanical systems are generally appearing into class of the oscillatory systems. Axially translating systems, for example, are appearing in such category. Axially translating systems have received much research attention since last six decades. Axially translating systems have been observed in many practical and engineering applications. The energy dissipation, known as damping, can easily be associated to axially translating systems, see Refs.[1-3]. Axially translating systems have many engineering applications. For example, conveyor belt systems, as given in Refs. [4-6], magnetic tapes, pipes conveying fluids, data saving devices, and elevator cable systems, see Ref. [5], and all such kind of systems are bound to vibrations. The study of axially translating systems with constant or time-varying velocity with viscous, material or boundary damping have received much importance in manufacture and design. It is common experience that the vibration causes severe failures to many mechanical or physical structures. From this viewpoint, it becomes necessary to design systems where unnecessary noise and vibrations can be reduced by means of solid procedures. Tacoma Narrows bridge is a good example being taught in education and research institutes for a complete structural collapse. This collapse was due to winds at certain speed. Apart from damaging structures, the vibration also causes human problems, that is, vibrations create anxiety to society. Keeping in view the effects of vibrations, it is matter of necessity to formulate methods and devise procedures to decrease vibrations from the physical and mechanical systems.

In several cases, damping devices can be kept through the support conditions to control vibrations through boundaries as seen in Refs. [4-7]. In Ref. [8] the damping device is introduced through whole spatial domain of the translating system. The reflection and damping properties for a wave equation have been studied in Ref. [9], where the authors have provided interesting results for a semi-infinite string. For different boundary conditions, in Ref. [10], the authors have provided detailed analysis for the energetics of the elevator cable systems. The authors, in Ref. [11], studied the energetics of an axially translating continuum, where they studied the case for fixed supports for string-like problem and the case for simple supports in case of beam-like problem. In Ref. [12], authors have provided detailed analysis of dampers connected at middle of string as well as beam. But the position of the damper always plays a significant role. If the damper is introduced at wrong spatial position it may increase energy of motion and may destabilize the system, for details see Ref. [13].

In this article, a materially damped beam-type equation has been considered. The paper is organized in the following way. In Sec. 2 the governing equations of motions are formulated based upon the Hamilton's principle. In Sec. 3 the analytic approximations of the solutions of an initial-boundary value problem have been obtained by using method of multiple timescales. Sec. 4 discusses results obtained in Sec. 3, and Sec. 5 represents the concluding remarks and directions for future research. 


\section{The Governing Equations}

The mathematical model of a traveling tensioned beam under material damping is based upon the following assumptions: $u(x, t)$ models the displacement field in vertical direction from equilibrium position, the mass of beam per unit length, $\rho$, is constant, the material damping coefficient $\bar{\beta}$ is constant, and the effects of gravity and other forces are neglected. Thus, the beam-type equation under material damping is given as follows,

$$
\begin{aligned}
\rho\left(u_{t t}+2 \bar{V} u_{x t}+\right. & \left.\dot{\bar{V}} u_{x}+\bar{V}^{2} u_{x x}\right)-T u_{x x}+E I u_{x x x x}+\bar{\beta}\left(u_{x x x x t}+\bar{V} u_{x x x x x}\right)=0 ; 0<x<L, t \\
& >0
\end{aligned}
$$

With simply supported boundary conditions,

and the general initial conditions,

$$
u(0, t)=u_{x x}(0, t)=u(L, t)=u_{x x}(L, t)=0 ; t>0
$$

$$
u(x, 0)=f(x), \text { and } u_{t}(x, 0)=g(x) ; 0<x<L .
$$

The terms in the bracket into Eq. (1) represent acceleration quantities. The equations contained in (1)-(3) can be put into a non-dimensional form by using following dimensionless quantities:

$u^{*}=\frac{u}{L}, x^{*}=\frac{x}{L}, t^{*}=\frac{c t}{L}, V_{0}=\frac{\bar{V}}{c}, \mu=\frac{E I}{\rho c^{2} L^{2}}, \delta_{0}=\frac{\bar{\beta}}{\rho c L^{3}}, f^{*}=\frac{f}{L}, g^{*}=\frac{g}{c}$, where $\quad c=\sqrt{T / \rho} \quad$ is $\quad$ the $\quad$ wave velocity.Thus, the equations (1)-(3) into non-dimensional form become:

$$
\begin{gathered}
u_{t t}+2 V_{0} u_{x t}+\dot{V}_{0} u_{x}+\left(V_{0}^{2}-1\right) u_{x x}+\mu u_{x x x x}+\delta_{0}\left(u_{x x x x t}+V_{0} u_{x x x x x}\right)=0 ; \\
0<x<1, t \geq 0 .
\end{gathered}
$$

The boundary conditions are given as,

$$
u(0, t)=u_{x x}(0, t)=0, \text { and } u(1, t)=u_{x x}(1, t)=0 ; t \geq 0 .
$$

The initial conditions are given as,

$$
u(x, 0)=f(x), \text { and } u_{t}(x, 0)=g(x) ; 0<x<1
$$

\section{The Analytic Approximations}

In this section an approximation of the solutions to the IBVP (4)-(6) by using a multiple timescales perturbation method is constructed. For a complete overview of this method, see Refs. [14-16]. Following two assumptions are made to utilize a this method. The velocity $\bar{V}$ of the beam is assumed to be small compared to wave velocity $c$ and that the damping coefficient $\bar{\beta}$ is small compared to $\rho c L^{3}$. Thus, it is reasonable to write $V_{0}=\frac{\bar{V}}{c}=O(\varepsilon)$, and $\delta_{0}=\frac{\bar{\beta}}{\rho c L^{3}}=O(\varepsilon)$, that is, $V_{0}=\varepsilon V$ and $\delta_{0}=\varepsilon \delta$. The parameter $\varepsilon$ is a dimensionless small parameter as described by $0<\varepsilon \ll 1$. Utilizing these assumptions in Eqs. (4)-(6), it follows that

$$
\begin{gathered}
u_{t t}-u_{x x}+\mu u_{x x x x}=-2 \varepsilon V u_{x t}-\varepsilon^{2} V^{2} u_{x x}-\varepsilon \delta u_{x x x x t}-\varepsilon^{2} \delta V u_{x x x x x}, \\
u(0, t ; \varepsilon)=u_{x x}(0, t ; \varepsilon)=0, \text { and } u(1, t ; \varepsilon)=u_{x x}(1, t ; \varepsilon)=0, \\
u(x, 0 ; \varepsilon)=f(x), \text { and } u_{t}(x, 0 ; \varepsilon)=g(x) .
\end{gathered}
$$

According to a two timescales method a function $u(x, t ; \varepsilon)$ is supposed to be a function of spatial variable $x$, the fast timescale $t=t$ and, the slow timescale $\tau=\varepsilon t$. For this reason,

$$
u(x, t ; \varepsilon)=y(x, t, \tau ; \varepsilon)
$$

By using Eq. (10), the time derivatives can be transformed as follows,

$$
\begin{gathered}
u_{t}=y_{t}+\varepsilon y_{\tau} \\
u_{t t}=y_{t t}+2 \varepsilon y_{t \tau}+\varepsilon^{2} y_{\tau \tau} .
\end{gathered}
$$

By substituting Eqs. (10)-(11) into Eqs. (7)-(9), the problem in yup to $O(\varepsilon)$ is given as follows,

$$
\begin{gathered}
y_{t t}-y_{x x}+\mu y_{x x x x}=-2 \varepsilon y_{t \tau}-2 \varepsilon V y_{x t}-\varepsilon \delta y_{x x x x t}, \\
y(0, t, \tau ; \varepsilon)=y_{x x}(0, t, \tau ; \varepsilon)=y(1, t, \tau ; \varepsilon)=y_{x x}(1, t, \tau ; \varepsilon)=0, \\
y(x, 0,0 ; \varepsilon)=f(x), y_{t}(x, 0,0 ; \varepsilon)=g(x)-\varepsilon y_{\tau}(x, 0,0 ; \varepsilon) .
\end{gathered}
$$

It is assumed that not onlyu $(x, t ; \varepsilon)$ can be approximated by the asymptotic expansion, but alsou $(x, t ; \varepsilon)=$ $y(x, t, \tau ; \varepsilon)$ can be approximated in the powers of $\varepsilon$ in the asymptotic expansion as,

$$
y(x, t, \tau ; \varepsilon)=y_{0}(x, t, \tau)+\varepsilon y_{1}(x, t, \tau)+\varepsilon^{2} \cdots,
$$

and that all the $y_{j}$ 's for $j=0,1,2, \cdots$, are found in such a way that no unbounded (secular) terms arise. It is also assumed that the unknown functions $y_{j}$ are $O(1)$. Now, by substituting Eq. (13) and its subsequent derivatives into Eq. (12), then by equating the powers of $\varepsilon^{0}$ and $\varepsilon^{1}$, and neglecting the $\varepsilon^{2}$ and the higher powers of $\varepsilon$, the $O(1)$-problem is followed as given by,

$$
\begin{aligned}
& y_{0_{t t}}-y_{0_{x x}}+\mu y_{0_{x x x x}}=0, \\
& y_{0}(0, t, \tau)=y_{0_{x x}}(0, t, \tau)=y_{0}(1, t, \tau)=y_{0_{x x}}(1, t, \tau)=0 \text {, }
\end{aligned}
$$




$$
y_{0}(x, 0,0)=f(x), y_{0_{t}}(x, 0,0)=g(x) .
$$

The $O(\varepsilon)$-problem is given as,

$$
\begin{gathered}
y_{1_{t t}}-y_{1_{x x}}+\mu y_{1_{x x x x}}=-2 y_{0_{t \tau}}-2 V y_{0_{x t}}-\delta y_{0_{x x x x t}}, \\
y_{1}(0, t, \tau)=y_{1_{x x}}(0, t, \tau)=y_{1}(0, t, \tau)=y_{1_{x x}}(1, t, \tau)=0, \\
y_{1}(x, 0,0)=0, y_{1_{t}}(x, 0,0)=-y_{0_{\tau}}(x, 0,0) .
\end{gathered}
$$

It can be observed that the $O(1)$-problem has solution only for the positive eigenvalues, $\lambda=\lambda_{n}=n^{2} \pi^{2}(1+$ $\left.n^{2} \pi^{2} \mu\right), n \in \mathbb{N}$, Ref. [17,18]. Thus, the solution of $O(1)$-problem is given as,

$$
y_{0}(x, t, \tau)=\sum_{n=1}^{\infty}\left(A_{n 0}(\tau) \cos \left(\sqrt{\lambda_{n}} t\right)+B_{n 0}(\tau) \sin \left(\sqrt{\lambda_{n}} t\right)\right) \phi_{n}(x),
$$

where $\phi_{n}(x)=\sin \left(\gamma_{n} x\right)-\frac{\sin \left(\gamma_{n}\right)}{\sinh \left(\beta_{n}\right)} \sinh \left(\beta_{n} x\right)$ are the eigenfunctions with $\gamma_{n}=\sqrt{\frac{-1+\sqrt{4 \lambda_{n} \mu+1}}{2 \mu}}$, and $\beta_{n}=$ $\sqrt{\frac{1+\sqrt{4 \lambda_{n} \mu+1}}{2 \mu}}$, and where $A_{n 0}$ and $B_{n 0}$ are undetermined functions. They can be obtained from the $O(\varepsilon)$ problem. The values of the constants $A_{n 0}(0)$ and $B_{n 0}(0)$ can easily be obtained by the initial values as given in Eq. (14) and by using the orthogonality properties of the eigenfunctions. The eigenfunctions $\phi_{p}(x), p \in \mathbb{N}$ satisfy the following orthogonality properties,

$$
\begin{aligned}
\int_{0}^{1} \phi_{p}(x) \phi_{q}(x) d x & =0, \text { for } p \neq q \\
& \neq 0, \text { for } p=q .
\end{aligned}
$$

Thus, by using the initial values as given in Eq. (14) and the orthogonality properties of the eigenfunctions as given in Eq. (17), $A_{n 0}$ and $B_{n 0}$ are given as

$$
\begin{gathered}
A_{n 0}(0)=\frac{\int_{0}^{1} f(x) \phi_{n}(x) d x}{\int_{0}^{1} \phi_{n}^{2}(x) d x}, \\
\sqrt{\lambda_{n}} B_{n 0}(0)=\frac{\int_{0}^{1} g(x) \phi_{n}(x) d x}{\int_{0}^{1} \phi_{n}^{2}(x) d x} .
\end{gathered}
$$

Now, the eigenfunction expansion method is introduced in solving the $O(\varepsilon)$-problem. Following form for the solution $y_{1}(x, t, \tau)$ is assumed,

$$
y_{1}(x, t, \tau)=\sum_{n=1}^{\infty} w_{n}(t, \tau) \phi_{n}(x)
$$

where $w_{n}(t, \tau)$ are the unknown functions of $\operatorname{tand} \tau$, and where $\phi_{n}(x)$ are the given eigenfunctions. Thus, by substitution of Eq. (20) in the $O(\varepsilon)$-equation, it yields

$$
\sum_{n=1}^{\infty}\left(w_{n_{t t}}(t, \tau)+\lambda_{n} w_{n}(t, \tau)\right) \phi_{n}(x)=-2 y_{0_{t \tau}}-2 V y_{0_{x t}}-\delta y_{0_{x x x x t}} .
$$

Now, by substitution of the solution $y_{0}(x, t, \tau)$ from Eq. (16) into Eq. (21), it follows that

$$
\begin{aligned}
\sum_{n=1}^{\infty}\left(w_{n_{t t}}(t, \tau)+\right. & \left.\lambda_{n} w_{n}(t, \tau)\right) \phi_{n}(x) \\
= & -2 \sum_{n=1}^{\infty} R_{n_{t \tau}}(t, \tau) \phi_{n}(x)-2 V \sum_{n=1}^{\infty} R_{n_{t}}(t, \tau) \phi_{n}^{\prime}(x) \\
& -\delta \sum_{n=1}^{\infty} R_{n_{t}}(t, \tau) \phi_{n}^{(i v)}(x)
\end{aligned}
$$

where $R_{n}(t, \tau)$ is given by,

$$
R_{n}(t, \tau)=A_{n 0}(\tau) \cos \left(\sqrt{\lambda_{n}} t\right)+B_{n 0}(\tau) \sin \left(\sqrt{\lambda_{n}} t\right) .
$$

By multiplying both sides of Eq. (22) with $\phi_{m}(x)$, then by integrating so-obtained equation from $x=0$ to $x=$ 1 with application of the orthogonality property of eigenfunctions, it follows that

$$
\begin{aligned}
& w_{m_{t t}}(t, \tau)+\lambda_{m} w_{m}(t, \tau) \\
& =-2 R_{m_{t \tau}}-2 V R_{m_{t}} \Theta_{m m}-\delta R_{m_{t}} \Delta_{m m}-\sum_{n=1, n \neq m}^{\infty}\left(2 V R_{n_{t}} \Theta_{n m}+\delta R_{n_{t}} \Delta_{n m}\right),
\end{aligned}
$$


where $\Theta_{n m}$ and $\Delta_{n m}$ are constants depending on the indices $m$ and $n$, and their values are given as follows

$$
\begin{gathered}
\Theta_{n m}=\frac{\int_{0}^{1} \phi_{n}^{\prime}(x) \phi_{m}(x) d x}{\int_{0}^{1} \phi_{m}^{2}(x) d x}, \\
\Delta_{n m}=\frac{\int_{0}^{1} \phi_{n}^{(i v)}(x) \phi_{m}(x) d x}{\int_{0}^{1} \phi_{m}^{2}(x) d x} .
\end{gathered}
$$

Note that $\Theta_{m m}=0$ for all integers $m$, whereas $\Delta_{m m}=\int_{0}^{1} \phi_{n}^{\prime \prime 2}(x) d x>0$. Thus, by using this value of $\Theta_{m m}$, changing index from $m$ to $n$ and making use of Eq. (23) into Eq. (24), it readily follows that

$$
\begin{aligned}
w_{n} t t & +\lambda_{n} w_{n}=\sqrt{\lambda_{n}}\left(2 A_{n 0}^{\prime}(\tau)+\delta \Delta_{n n} A_{n 0}(\tau)\right) \sin \left(\sqrt{\lambda_{n}} t\right) \\
& -\sqrt{\lambda_{n}}\left(2 B_{n 0}^{\prime}(\tau)+\delta \Delta_{m n} B_{n 0}(\tau)\right) \cos \left(\sqrt{\lambda_{n}} t\right) \\
& +\sum_{m=1, m \neq n}^{\infty}\left\{\left(2 V \Theta_{m n}+\delta \Delta_{m n}\right) \sqrt{\lambda_{m}} A_{m 0}(\tau) \sin \left(\sqrt{\lambda_{m}} t\right)\right. \\
& \left.-\left(2 V \Theta_{m n}+\delta \Delta_{m n}\right) \sqrt{\lambda_{m}} B_{m 0}(\tau) \cos \left(\tau \sqrt{\lambda_{m}} t\right)\right\}
\end{aligned}
$$

On right hand side of Eq. (26) first two terms are solutions of the homogeneous equation. Such terms will give rise to secular terms in $w_{n}(t, \tau)$. Since it is assumed that the functions $y_{0}(x, t, \tau), y_{1}(x, t, \tau), \cdots$ are bounded on timescale of $O\left(\varepsilon^{-1}\right)$. Thus, to have secular free behavior, the following solvability conditions are imposed in Eq. (26),

$$
\begin{aligned}
& A_{n 0}^{\prime}(\tau)+\Delta_{n n} \frac{\delta}{2} A_{n 0}(\tau)=0, \\
& B_{n 0}^{\prime}(\tau)+\Delta_{n n} \frac{\delta}{2} B_{n 0}(\tau)=0 .
\end{aligned}
$$

The solutions to above system of two uncoupled ordinary differential equations in (27) are given as follows

$$
\begin{aligned}
& A_{n 0}(\tau)=A_{n 0}(0) \mathrm{e}^{-\Delta_{n n} \frac{\delta}{2} \tau}, \\
& B_{n 0}(\tau)=B_{n 0}(0) \mathrm{e}^{-\Delta_{n n} \frac{\delta}{2} \tau},
\end{aligned}
$$

where $A_{n 0}(0)$ and $B_{n 0}(0)$ are given in Eqs. (18) and (19), respectively. Thus, by using Eq. (28) into Eq. (16), the complete solution to the $O(1)$-problem is given as follows,

$$
y_{0}(x, t, \tau)=\sum_{n=1}^{\infty} \mathrm{e}^{-\Delta_{n n} \frac{\delta}{2} \tau}\left(A_{n 0}(0) \cos \left(\sqrt{\lambda_{n}} t\right)+B_{n 0}(0) \sin \left(\sqrt{\lambda_{n}} t\right)\right) \phi_{n}(x) .
$$

Now, by substituting $\tau=\varepsilon t$ into the expression, $-\Delta_{n n} \frac{\delta}{2} \tau$, and then dividing the so-obtained expression by $t$, the damping parameter $\Gamma_{n}$ for all the oscillation modes can be approximated by,

From Eq. (26) with Eq. (27), it follows that

$$
\Gamma_{n}=-\varepsilon \Delta_{n n} \frac{\delta}{2} .
$$

$$
\begin{aligned}
w_{n t t}+(n \pi)^{2} w_{n} & =\sum_{\substack{m=1, m \neq n\\
}}^{\infty}\left(2 V \Theta_{m n}+\delta \Delta_{m n}\right) \sqrt{\lambda_{m}}\left(A_{m 0}(\tau) \sin \left(\sqrt{\lambda_{m}} t\right)\right. \\
& \left.-B_{m 0}(\tau) \cos \left(\sqrt{\lambda_{m}} t\right)\right) .
\end{aligned}
$$

Now, it can be seen that the Eq. (31) is a second order nonhomogeneous ODE in $w_{n}(t, \tau)$. Thus, the solution to Eq. (31) is given as

$$
\begin{gathered}
w_{n}(t, \tau)=A_{n 1}(\tau) \cos \left(\sqrt{\lambda_{n}} t\right)+B_{n 1}(\tau) \sin \left(\sqrt{\lambda_{n}} t\right) \\
+\sum_{m=1, m \neq n}^{\infty} \frac{\left(2 V \Theta_{m n}+\delta \Delta_{m n}\right) \sqrt{\lambda_{m}}}{\lambda_{n}-\lambda_{m}}\left(A_{m 0}(\tau) \cos \left(\sqrt{\lambda_{m}} t\right)\right. \\
\left.-B_{m 0}(\tau) \sin \left(\sqrt{\lambda_{m}} t\right)\right),
\end{gathered}
$$

where $A_{n 1}(\tau)$ and $B_{n 1}(\tau)$ areyet undetermined functions of a slow variable $\tau$, these functions can be obtained from the $O\left(\varepsilon^{2}\right)$-problem. Thus, the Eq. (20) with Eq. (32) can be expressed as 


$$
\begin{aligned}
y_{1}(x, t, \tau)=\sum_{n=1}^{\infty}\{ & A_{n 1}(\tau) \cos \left(\sqrt{\lambda_{n}} t\right)+B_{n 1}(\tau) \sin \left(\sqrt{\lambda_{n}} t\right) \\
& +\sum_{m=1, m \neq n}^{\infty} \frac{\left(2 V \Theta_{m n}+\delta \Delta_{m n}\right) \sqrt{\lambda_{m}}}{\lambda_{n}-\lambda_{m}}\left(A_{m 0}(\tau) \cos \left(\sqrt{\lambda_{m}} t\right)\right. \\
& \left.\left.-B_{m 0}(\tau) \sin \left(\sqrt{\lambda_{m}} t\right)\right)\right\} \phi_{n}(x) .
\end{aligned}
$$

Now, by using the inner product (17) and the initial values in Eq. (15) with Eq. (16) into Eq. (33), it follows that $A_{n 1}(0)$ and $B_{n 1}(0)$ are given by

$$
\begin{aligned}
A_{n 1}(0) & =-\sum_{m=1, m \neq n}^{\infty} \frac{\left(2 V \Theta_{m n}+\delta \Delta_{m n}\right) \sqrt{\lambda_{m}}}{\lambda_{n}-\lambda_{m}} A_{m 0}(0), \\
\sqrt{\lambda_{n}} B_{n 1}(0) & =\sum_{m=1, m \neq n}^{\infty} \frac{\left(2 V \Theta_{m n}+\delta \Delta_{m n}\right) \lambda_{m}}{\lambda_{n}-\lambda_{m}} B_{m 0}(0)-A_{n 0}^{\prime}(0) .
\end{aligned}
$$

It can be seen that the solution $y_{1}(x, t, \tau)$ still contains infinitely many undetermined functions $A_{n 1}(\tau)$ and $B_{n 1}(\tau)$, for $n \in \mathbb{N}$. These unknown functions can be used to avoid secular terms in solution $y_{2}(x, t, \tau)$. At this time, it is not reasonable to construct the higher order calculations. This is a reason, we can take $A_{n 1}(\tau)=A_{n 1}(0)$ and $B_{n 1}(\tau)=B_{n 1}(0)$. So far, a asymptotic expansion $y(x, t, \tau)=y_{0}(x, t, \tau)+$ $\varepsilon y_{1}(x, t, \tau)$ has been constructed for $u(x, t)$.

\section{Results and Discussion}

This section is all about to comment, to interpret and to explain the results obtained in Sec. 3. Using the complete analytical solution of the $O(1)$-problem, the influence of small parameter $\varepsilon$ and the damping parameter on the axially moving system will be discussed in detail. By using the $O(1)$-solution and the $O(\varepsilon)$ solution, it yields

$$
u(x, t)=\sum_{n=1}^{\infty} \mathrm{e}^{-\Delta_{n n} \frac{\delta}{2} \varepsilon t}\left(A_{n 0}(0) \cos \left(\sqrt{\lambda_{n}} t\right)+B_{n 0}(0) \sin \left(\sqrt{\lambda_{n}} t\right)\right) \phi_{n}(x),
$$

where $A_{n 0}(0) \operatorname{and} B_{n 0}(0)$ are given by Eqs. (18) and (19). From physical point of view, all terms can be explained in above solution to the IBVP (4)-(6).The terms $A_{n 0}(0) \cos \left(\sqrt{\lambda_{n}} t\right)+B_{n 0}(0) \sin \left(\sqrt{\lambda_{n}} t\right)$ arethe oscillation terms obtained from a time-dependent part of the equation. These terms oscillate with frequencies $n \pi \sqrt{1+n^{2} \pi^{2} \mu}$ for $n \in N$. The term $\mathrm{e}^{-\Delta_{n n} \frac{\delta}{2} \varepsilon t}$ is occurring due to material damping. This term shows that as the time parameter $t$ will increase for fixed values of $\delta$ and $\varepsilon$ the size of the oscillation amplitudes $A_{n 0}(0)$ and $B_{n 0}(0)$ will start to decrease and it is also shown that as mode number $n$ starts to increase the oscillation amplitudes tend to decrease for fixed $\delta, \varepsilon$, and $t$. The last term $\phi_{n}(x)$ is the solution of the space-dependent part which describes the shapes of the oscillation curves along $x$-axis.

\section{Conclusions and Future Work}

In this paper, an initial-boundary value problem (IBVP) for the materially damped axially translating continuum has been studied. Solving the IBVP a method of two timescales has successfully been applied to obtain the analytic solutions of a mathematical model which describes the transversal vibrations of a conveyor belt system. It has been shown, in this paper, that all oscillation modes are damped for the system. It has also been shown that damping rates are, in fact, depending on mode numbers $n$. From mechanical point of view, this response is reasonable because as oscillations increase the more heat is generated in the system which internally damps the vibratory energy of motion. As modes increase the oscillation amplitudes decrease and the belt system gets stable. This research problem can further be extended to internal damping of an axially translating string as well as axially translating beam with time-dependent axial velocities.

\section{References}

[1]. Darmawijoyo, van Horssen WT. On the Weakly Damped Vibrations of a String Attached to a Spring-Mass-Dashpot System. Journal of Vibration and Control 2003;9:1231-1248.

[2]. Darmawijoy, van Horssen WT. On Boundary Damping for a Weakly Nonlinear Wave Equation. Nonlinear Dynamics 2002;30:179191.

[3]. Darmawijoyo, van Horssen WT. On a Rayleigh Wave Equation with Boundary Damping. Nonlinear Dynamics 2003;33(4):399-429.

[4]. Sandilo SH, van Horssen WT. On Boundary Damping for an Axially Moving Beam and On Variable Length Induced Oscillations of an Elevator Cable. In: Proceeding of the 7th European Nonlinear Dynamics Conference. Rome, Italy: 2011. p. 1-6. 
[5]. Sandilo SH, van Horssen WT. On Boundary Damping for an Axially Moving Tensional Beam. Journal of Vibration and Acoustics 2012;134:0110051-8.

[6]. Gaiko N, van Horssen WT. On the Transverse, Low Frequency Vibrations of a Travelling String with Boundary Damping. Journal of Vibration and Acoustics 2015;DOI: 10.1115/1.4029690.

[7]. Zarubinskaya MA, van Horssen WT. On Aspects of Boundary Damping for a Rectangular Plate. Journal of Sound and Vibration 2006;292:844-853.

[8]. Maitlo AA, Sandilo SH, Sheikh AH, Malookani RA, Qureshi S. On Aspects of Viscous Damping for an Axially Transporting String. Science International (Lahore) 2016;28:3721-3727.

[9]. Akkaya T, van Horssen WT. Reflection and Damping Properties for Semi-Infinite String Equations with Non-Classical Boundary Conditions. Journal of Sound and Vibration 2015;336:179-190.

[10]. Zhu WD, Ni J. Energetics and Stability of Translating Media with an Arbitrary Varying Length. Journal of Vibration and Acoustics 2000;122:295-304

[11]. Wickert JA, Mote CD. On the Energetics of an Axially Moving Continua. Journal of Acoustical Society of America 1989;85:13651368.

[12]. Main JA, Jones NP. Vibration of Tensioned Beams with Intermediate Damper. I: Formulation, Influence of Damper Location. Journal of Engineering Mechanics 2007;133:369-378.

[13]. Hegedorn P, Seemann W. Modern Analytical Methods Applied to Mechanical Engineering Systems: Part6 in Modern Methods of Analytical Mechanics and Their Applications. In: Rumyantsev VV, Karaptyan AV, Editors. CISM Courses and Lecturers no. 389, Springer-Verlage, Wien New York, 1998. p. 317-328.

[14]. Nayfeh AH. Introduction to Perturbation Techniques. Wiley-VCH Verlag GmbH and Co, 2004.

[15]. Kevorkian J, Cole JD. Multiple Scale and Singular Perturbation Methods. Springer-Verlag, New York, 1996.

[16]. Murdock JA. Perturbation: Theory and Methods. Classics in Applied Mathematics, SIAM, 1999.

[17]. Haberman R. Applied Partial Differential Equations with Fourier Series and Boundary Value Problems. Pearson, $5^{\text {th }}$ edition, 2012.

[18]. Hagedorn P, DasGupta A. Vibrations and Waves in Continuous Mechanical Systems. John Wiley and Sons, 2007. 